\title{
Definición de un ROLE I sobre contenedor «6 en I" de la Unidad Médica Aérea de Apoyo al Despliegue (UMAAD) Zaragoza
}

\author{
Laguardia Chueca, JC. ${ }^{1}$, Díez Cruz, A. ${ }^{1}$, Rodrigo Arrastio CF. ${ }^{2}$, Pérez Rodríguez CA. ${ }^{3}$, Fernández Peña MD. ${ }^{4}$, \\ Alcheikh Felices, MM. ${ }^{5}$
}

Sanid. mil. 2012; 68 (1): 40-43; ISSN: 1887-8571

\begin{abstract}
RESUMEN
Introducción: La Unidad Médica Aérea de Apoyo al Despliegue (UMAAD) de Zaragoza posee un contenedor ISO de 20 pies, expandible (6 en 1) para su uso como Role 1 (Atención primaria y de Urgencia) dentro del despliegue de un destacamento del Ejército del Aire. Material: Definición de la disposición interna, sistemas auxiliares y material de un Role 1 montado sobre un contenedor 6 en 1. Funcionamiento: El contenedor 6 en 1 de la UMAAD, es un elemento de gran utilidad, versatilidad y funcionalidad que permite emplazar, en un espacio limitado y en muy poco tiempo, una Instalación de Tratamiento Médico con capacidad Role 1 con elevado potencial de transformación interna, lo cual resulta extremadamente útil para garantizar el nivel asistencial adecuado en Atención Primaria y de Urgencias para el personal desplegado en una misión. El tiempo de despliegue y montaje del contenedor, en dependencia de la orografía del suelo, puede oscilar entre 30 y 120 minutos, y la instalación del material y sistemas auxiliares, necesario para ser operativo en unas 2-4 horas. El personal que se precisaría para este montaje no supera las 6 personas. Además dicho material puede ser trasportado por vía terrestre, aérea y marítima lo que lo hace muy adecuado para su uso en el medio militar. Conclusiones: El uso de un contenedor 6 en 1 para su utilización como puesto de Atención Primaria y Urgencias (Role 1), mejora la calidad de la atención al paciente y amplía la capacidad de servicios que se pueden realizar en operaciones de proyección del personal del Ejército del Aire y, en general, de las Fuerzas Armadas.
\end{abstract}

PALABRAS CLAVE: Role 1, Contenedor, UMAAD Zaragoza, Unidad médica, Apoyo Sanitario, Despliegue.

\section{Definition Role 1 in container 6 to 1 from UMAAD Zaragoza}

SUMMARY

Introduction: Air Medical Deployment Support Unit (UMAAD) of Zaragoza has a 20-feet ISO container, expandable (6 in 1) to use as a Role 1 (Primary Care and Emergency) in the deployment of any detachment of Spanish Air Force. Material: Definition of internal arrangement, auxiliary systems and supplies for a Role 1 mounted on a 6 in 1 container. Functions: The UMAAD's 6 in 1 container is an element of great utility, versatility and functionality in order to have enough space in a very short time to deploy as a Role 1, with powerful inner transformation that makes it very useful for primary care and emergency staff deployed in a mission. Deployment time and installation of the container, depending on the topography of the soil, can vary between 30 and 120 minutes, and the installation of equipment and auxiliary systems required to be operational in about 2-4 hours. Personnel required for this installation would be about six people. Also this material can be transported by land, sea and air making it very suitable for its use by the military. Conclusions: The 6 in 1 container usage as a post of Primary Care and Emergency (Role 1) improves the quality of patient care and extends the function capability that can be performed on projected operations staff of the Air Force and, in general, Armed Forces.

KEY WORDS: Role 1, Container, UMAAD Zaragoza, Medical Unit, Health Support, Deployment.

\section{INTRODUCCIÓN}

La Unidad Médica Aérea de Apoyo al Despliegue (UMAAD) de Zaragoza se encuentra encuadrada orgánicamente en el Mando Aéreo de Combate del Ejército del Aire, y cuenta en su dotación con un contenedor 6 en 1, que ha sido adaptado para su utilización como Role 1, dentro de las responsabilidades de Apoyo Sanitario que la

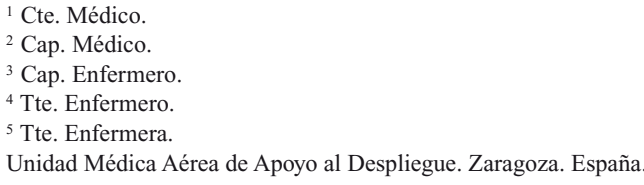

Dirección para correspondencia: Juan Carlos Laguardia Chueca. UMAAD. Base Aérea de Zaragoza. Carretera del Aeropuerto s/n. 50071 Zaragoza. Email: jlagchu@ea.mde.es

Recibido: 30 de septiembre de 2010

Aceptado: 31 de octubre de 2011
Instrucción 6/2003 del Jefe del Estado Mayor del Aire ${ }^{1}$, por la que se crea la Unidad, le asigna. Este Apoyo Sanitario a las Fuerzas Desplegadas $^{2}$, se podrá proporcionar a su vez, en casos de catástrofe natural o de misiones de Ayuda Humanitaria, reconstrucción o desarrollo ${ }^{3-5}$.

\section{Definición Role 1}

El concepto de Role 1, como categoría del Apoyo Sanitario, lo encontramos, originalmente, en los documentos doctrinales de OTAN. En primer lugar se nos presenta en uno de los documentos del Comité Militar de OTAN, el NATO MC (Military Commitee ) 326/2, que trata sobre «Principles and Policies of Operational Medical Support». Este documento tiene como cometido ser una guía para el desarrollo compatible entre las distintas naciones sobre los conceptos, planes y estructuras del soporte médico. Define el Role 1 como «medical support provides for routine primary health care, specialized first aid, triage, resuscitation and stabilization $»^{6}$. 


\section{Definición de un ROLE 1 sobre contenedor «6 en 1» de la Unidad Médica Aérea de Apoyo al Despliegue...}

La AJP 4-10 «Allied Joint Medical Support Doctrine»7, incluye el Role 1 dentro de las Medical Treatment Facilities (MTFs) desplegables. Estas se definen como unidades de las mismas características de movilidad y protección que las unidades a las que apoyan encargadas de proveer de cuidados médicos, en el lugar más apropiado y cercano a la zona de combate, en el menor tiempo posible.

La clasificación de los MTFs se realiza en función de las capacidades quirúrgicas disponibles en cada una de ellos. Así, el Role 1 carece de capacidad quirúrgica (salvo en lo referente a cirugía menor); el Role 2 dispone de Cirugía de Control del Daño y/o Cirugía General; el Role 3 cuenta con un número variable de especialidades quirúrgicas, y el Role 4 (el único no desplegable y, normalmente localizado en Territorio Nacional) está dotado de todas las capacidades asistenciales para garantizar el tratamiento definitivo de las lesiones ${ }^{8}$.

Otra de las características del Role 1 es que generalmente es de responsabilidad nacional, a diferencia de los superiores (Role 2 y 3 ), los cuales conforman unidades independientes y en los que su composición puede ser multinacional ${ }^{9,10}$.

La Doctrina Sanitaria Conjunta asigna al Primer Escalón, dentro del capítulo 5 sobre el Escalonamiento Sanitario en Operaciones, la capacidad de «prestar apoyo sanitario inmediato a las bajas, de recogerlas y de realizar la clasificación inicial». Por otra parte considera que «el 1. ${ }^{\text {er }}$ Escalón de Apoyo Sanitario es orgánico o asignado a una unidad». De todas formas, conviene insistir en la no equivalencia completa y directa entre Role 1 y Primer Escalón Sanitario.

\section{Capacidades y cometidos de un Role 1}

Entre las distintas funciones que puede desarrollar un Role 1, podemos destacar:

1. La recogida de bajas, en el lugar más cercano posible donde se produjo (normalmente en un nido de heridos o vehículo), la evacuación sanitaria, su clasificación o triaje, la realización de las maniobras de soporte vital básico y avanzado, el tratamiento inicial, la estabilización y puesta en estado de evacuación de la baja. Tabla 1 .

2. Junto a estas funciones de tratamiento, incluye la filiación y control del personal que atiende, así como realizar las medidas preventivas pertinentes frente a enfermedades comunes y lesiones no de combate.

3. Puede disponer de una mínima capacidad de hospitalización, odontología y laboratorio básicos, así como medidas para el tratamiento y cuidado inicial del estrés de combate.

4. En el caso de que el Apoyo Sanitario se preste en el ámbito de Operaciones Aéreas (circunstancia particular de la UMAAD Zaragoza dada su naturaleza de Unidad del Ejército del Aire

Tabla 1. Capacidades de un ROLE 1.

\begin{tabular}{|ll|}
\hline Basicas & - \\
& Asesoramiento a la cadena de mando sobre Medicina \\
- & Tratamiento de patología y lesiones en Atención Primaria \\
& para la reincorporación inmediata al trabajo. \\
- & Recogida de heridos desde el punto de lesión \\
- & Preparación de Bajas para su evacuación a un nivel superior \\
& de MTF (Role 2, 3, 4). \\
Opcionales - & Mínima capacidad de hospitalización de pacientes. \\
- & Odontología de Atención Primaria. \\
- & Laboratorio básico mediante Test. \\
- & Tratamiento del estrés inicial.
\end{tabular}

encuadrada en el Mando Aéreo de Combate), se contempla la capacidad de Medicina de Vuelo ${ }^{12}$.

\section{MATERIAL}

La Dirección de Sanidad del Ejército del Aire asignó a la UMAAD Zaragoza un contendor aerotransportable, Norma ISO, 20 $\mathrm{ft}$ (dimensiones 20x8x8 ft).

Dicho contenedor, fabricado por la empresa Getelec (Francia) y distribuido en España por Europavía Ibérica, tiene una longitud de 20 pies, una anchura y altura de 8 pies (medidas métricas: 6,8 metros de largo por 2,45 $\mathrm{m}$ de ancho y 2,45 metros de altura), lo que posibilita su aerotransporte. Su peso es de $7.500 \mathrm{~kg}$.

Está diseñado para soportar cambios de temperatura que oscilan entre los $-20 \mathrm{y}+50^{\circ} \mathrm{C}$ y precipitaciones de lluvia de $100 \mathrm{~m}^{3} / \mathrm{h}$, y de nieve de $180 \mathrm{~kg} / \mathrm{m}^{2}$. Tabla 2 .

Para su funcionamiento precisa de una alimentación eléctrica externa de 380/400 V, trifásica y $50 \mathrm{~Hz}$.

Otra característica importante es que aloja en su interior cinco módulos más, de tal forma que, una vez extendido, alcanza los 13 metros de longitud por 6,8 metros de anchura y 2,45 metros de altura, lo que hace una superficie útil aproximada de $72 \mathrm{~m}^{2}$.

\section{FUNCIONAMIENTO}

\section{Áreas Funcionales}

Tras un estudio preliminar realizado, se preparó para su adaptación a una Instalación de Tratamiento Médico de Apoyo Sanitario con capacidad Role 1, y se definió su disposición interna, creando cuatro zonas diferenciadas:

\section{Admisión. Recepción}

Está situada en el primer compartimento y dispone de una superficie de unos $12 \mathrm{~m}^{2}$. Además del mobiliario específico al uso, se ubica en ella el equipamiento informático correspondiente, los sistemas de comunicaciones, telefonía y radio, estándar y de emergencia, así como el equipamiento sanitario para atender emergencias exteriores.

\section{Triaje, Atención Primaria, Odontología}

La sala, separada de la anterior, dispone de una superficie de unos $24 \mathrm{~m}^{2}$. Aloja el equipamiento asistencial específico de una sala

\section{Tabla 2. Especificaciones técnicas}

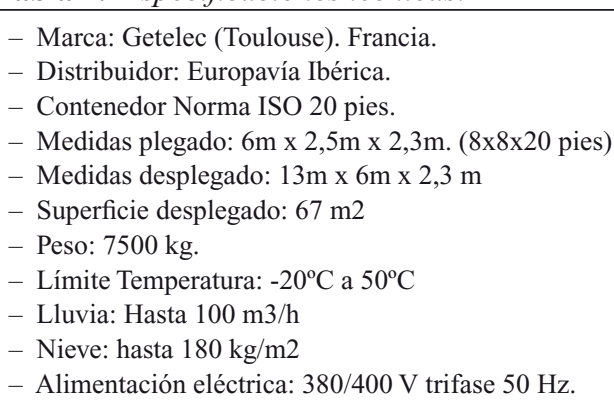




\section{J. C. Laguardia Chueca, et al.}

de Urgencias y Atención Primaria, con dos camillas de exploración, dotadas, cada una de ellas, de toma de oxígeno individual tanto para gafas nasales/mascarilla, como para respirador si llegase a ser preciso.

También se ubica en ella, el equipamiento específico del Médico de Vuelo y el propio de un laboratorio clínico. Este laboratorio desarrollaría pruebas básicas mediante técnicas de test.

Llegado el caso, se puede sustituir una de las camillas de exploración por un sillón odontológico, si las condiciones de la misión exigiesen disponer de dicha capacidad.

Respecto al equipamiento auxiliar, aloja el lavabo de agua corriente, la nevera de medicamentos y el autoclave para esterilización del equipamiento e instrumental de curas.

\section{Hospitalización. Telemedicina}

Esta área dispone de dos camas de hospitalización equipadas con respirador, monitor y bomba de perfusión. Ambas disponen, al igual que las camillas de triaje, de toma de oxígeno independiente tanto para gafas nasales o mascarilla, como para respirador. Figura 1.

En esta zona también se ubica el equipo de Telemedicina así como el carro de parada.

La superficie asignada a esta área es de $24 \mathrm{~m}^{2}$ aproximadamente.

\section{Secretaría. Dirección. Zona Técnica}

Es la zona correspondiente al contendor inicial y que aloja al resto cuando se repliega.

En ella se ubican dos puestos de oficina con su equipamiento informático, mobiliario de gestión y comunicaciones telefónicas.

La Zona Técnica es el punto de entrada del sistema de distribución de oxígeno y el sistema de control ambiental. Aloja el armario de red local y su conexión exterior, el cuadro y conexión exterior de telefonía así como el armario de limitadores y diferenciales eléctricos.

\section{Sistemas Auxiliares}

Para una óptima utilización del contenedor como MTF con capacidad de Role 1 se consideró necesario dotarlo de una serie de Sistemas Auxiliares que comprenden:

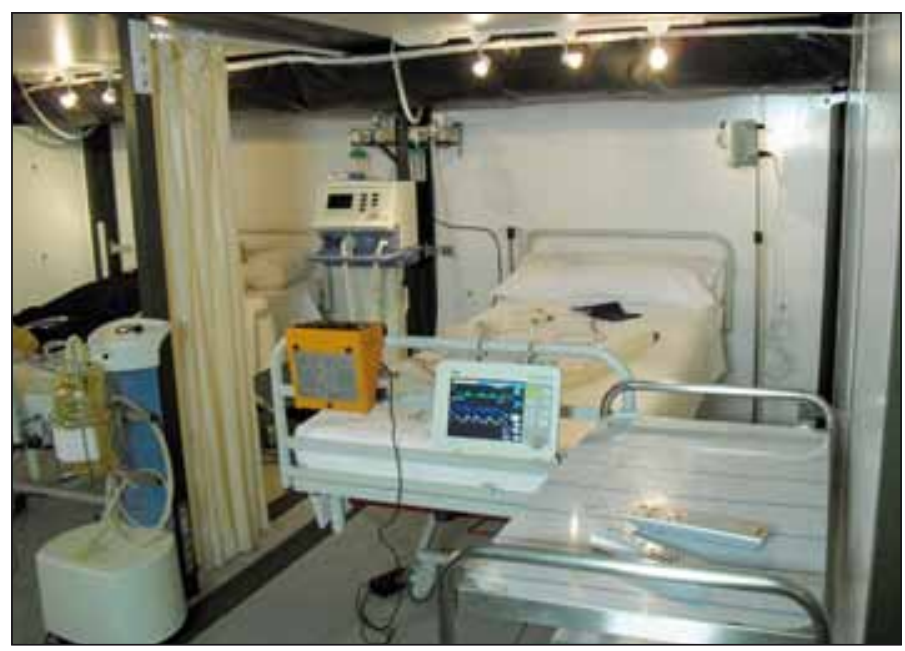

Figura 1. Área de Estabilización y Telemedicina.

\section{Sistema de Control Ambiental}

Se utiliza un equipo externo de frío (Zhendre) con una potencia frigorífica de $10.2 \mathrm{Kw}$, y un equipo de calor (Kroll) que proporciona $46 \mathrm{Kw}$ o $39.600 \mathrm{Kcal} / \mathrm{h}$. Fue necesario diseñar una adaptación para su conexión con el contenedor. El sistema de distribución interno se realiza mediante una vaina colocada por el techo, a lo largo de todo el contendor.

\section{Sistema de distribución de oxígeno}

El aporte se realiza desde una fuente externa (botellas o generador de $\mathrm{O}_{2}$ ), acoplada a la instalación por medio de un conector y se distribuye por un sistema de cable reforzado para oxígeno a 4 cajas con tomas dobles, para oxígeno convencional y para respirador ${ }^{13}$. Figura 2.

\section{Sistema eléctrico}

Se utilizó el sistema proveniente de fábrica (tomas de fuerza e iluminación: $220 \mathrm{~V}$ ), aunque hubo que añadir y modificar alguna de las tomas de fuerza distribuidas por el interior del contenedor. Como fuente externa se precisa de un generador o una conexión a la red local. La instalación original viene limitada a 45 Kva y la conexión se realiza mediante clavija de 5 pins de 32 Amp. Tabla 3.

\section{Sistema de agua corriente}

Precisa de un grupo de presión externo sobre una fuente de agua o depósito, así como un sistema de desagüe a sistema de vertidos locales o a un depósito de aguas residuales.

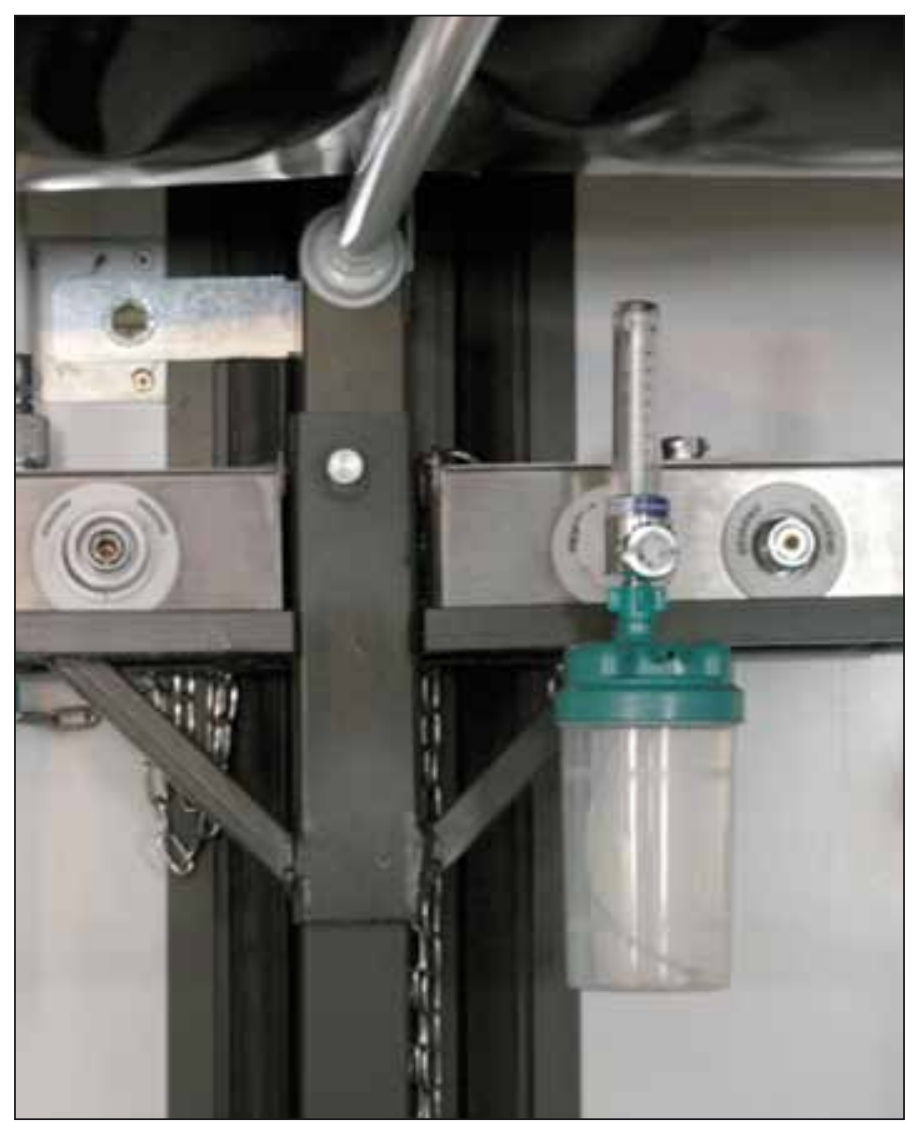

Figura 2. Tomas de Oxígeno: Respirador y Gafas nasales. 
Tabla 3. Sistemas auxiliares.

\begin{tabular}{|ll|}
\hline Climatización & - Equipo externo de frío y calor. \\
& - Distribución por vainas. \\
Oxigenoterapia & - Fuente externa: Botellas. Generador. \\
& - Distribución: 4 tomas dobles. \\
Electricidad & - Fuente externa: Generador/Red Local. \\
& - Limitador de $50 \mathrm{Kva}, 380 \mathrm{v}$. trifásica. \\
& - Conexión : Clavija 5 pins 32 Amp \\
& - Tomas de fuerza e iluminación: $220 \mathrm{v}$ \\
Distribución de agua & - Grupo de presión externo sobre fuente de agua. \\
& - Sistema de desagüe: Depósito de aguas residuales \\
Comunicaciones & - Red de Área Local (LAN) para datos. \\
& - Telefonía. \\
& - Comunicación satélite: Telemedicina \\
& - Radio \\
\hline
\end{tabular}

\section{Sistema de comunicaciones}

Se ha realizado, mediante recursos propios de la Unidad, las adaptaciones necesarias para incorporar los tres sistemas básicos de comunicaciones precisas.

- Telefonía: Central de distribución, cableado y conexiones de RJ11 en todas las áreas funcionales.

- Red de Área Local (LAN): Router, cableado y conexiones RJ45 en las distintas áreas funcionales

- Radio: Cableado para antena exterior en la zona de Recepción.

- Satélite: Adaptación para salida de antena de M4 del Equipo de Telemedicina (Cabe la posibilidad de conexión mediante Telefonía-Fleximus).

Con la modificación y adaptación de este contendor, la UMAAD Zaragoza se dota con la capacidad de desplegar un Role 1 sobre instalación rígida, cumpliendo todas las exigencias y cometidos que la doctrina OTAN (AJP-4.10) exige. Tabla 4.

\section{CONCLUSIONES}

El contenedor ISO 20 pies, desplegable 6 en 1 asignado a la UMAAD Zaragoza, proporciona a la Unidad la capacidad de Role 1 sobre estructura rígida a la vez que proyectable.

Es un elemento de gran interés desde el punto de vista logístico y operativo debido a su fácil montaje y desmontaje, su posibilidad de desplazamiento por distintos medios de transporte y su capacidad de transformación interior, dependiendo de las necesidades.

La incorporación de los sistemas auxiliares facilita la realización de la misión asistencial al integrar todas las tareas en una misma y única instalación.

La zonificación y el aislamiento relativo de cada área funcional permiten el proceso asistencial sin interferencias internas ni externas, garantiza la adecuada independencia y, finalmente, posibilita la realización simultánea de tareas diversas y no conectadas entre sí directamente.

Posibilita otros usos alternativos, por lo que podría utilizarse como una dependencia más de una instalación sanitaria tipo Role 2 o Role 3. En ambos casos puede ser destinado a uno de los siguientes cometidos: farmacia, sala de hospitalización, sala de vida y funcionamiento, etc. Figura 3.
Tabla 4. Equipamiento asistencial.

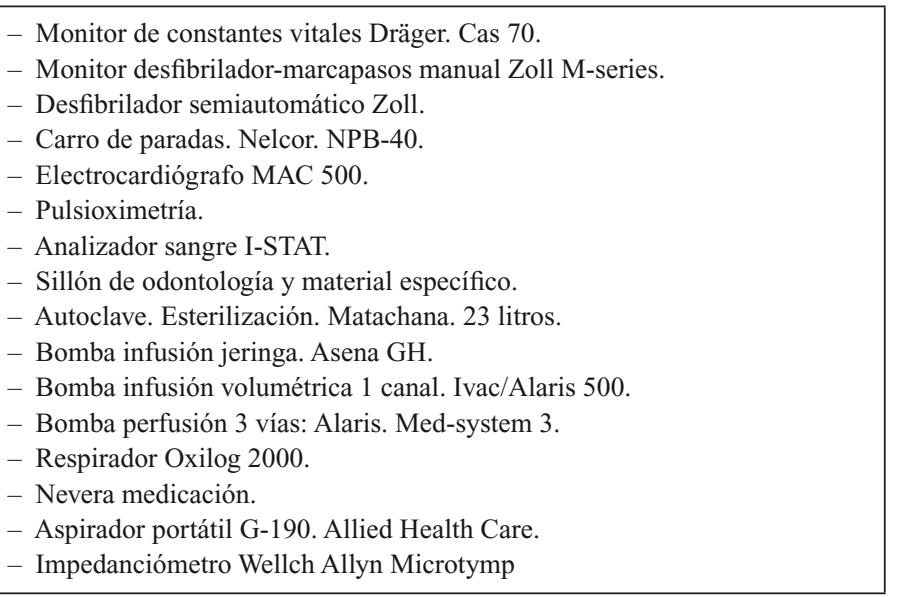

\section{BIBLIOGRAFÍA}

1. Instrucción General 6/2003 del Jefe del Estado Mayor del Aire

2. Bricknell DM. Roles for international military medical services in stability operations. JR Army Med Corps. 153(3): 160-164.

3. Sharp TW, Wightman JM, Davis MJ, Sherman SS, Burkle FM. Military assistance in complex emergencies: what have we learned since the Kurdish relief effort? Prehosp Disaster Med ; 16(4) :197-208.

4. Yeskey KS, Llewellyn CH, Vayer JS. Operational medicine in disasters. Emerg Med Clin North Am May 1996; 14(2) :429-38.

5. Bricknell MC, Gadd RD. Roles for international military medical services in stability operations (reconstruction and development). J R Army Med Corps Sep 2007; 153(3) :160-4.

6. MC 326/2: Nato Principles and Policies of Operational Medical Support. (2003)

7. A.J.P-4.10 (Allied Joint Medical Support Doctrine).

8. Hetz SP. Introduction to military medicine: a brief overview. Surg Clin North Am Jun 2006; 86(3) :675-88.

9. Klein L. The medical mission in NATO operations. Acta Chir Plast 2004; 46(2): 59-62.

10. Hazard L. Primary casualty receiving facility [Argus on Operational Sea Training]. J R Nav Med Serv 2005; 91(3):158-60

11. Alsina Álvarez J., Instalaciones Sanitarias Desplegables Multinacionales. Modelos de colaboración. Sanid. Mil.; 63 (4): 323-327.

12. The Air Force and the Medical Service. Can Med Assoc J Aug 1918; 8(8): 736-8.

13. P. Evrard. Production d'oxygène sur site appliquée aux hôpitaux de champagne. Science Direct. IRBM 28 (2007); 93-106.

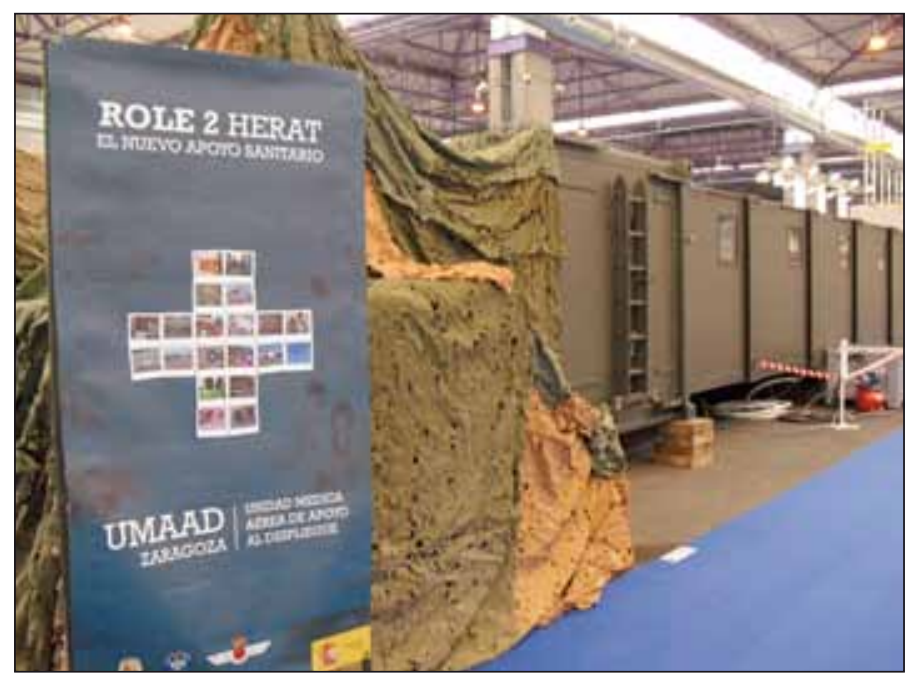

Figura 3. Vista Exterior del Contenedor Desplegado. 